\title{
NNK Metabolite
}

National Cancer Institute

\section{Source}

National Cancer Institute. NNK Metabolite. NCI Thesaurus. Code C129884.

Molecules created during the catabolism of the nitrosamine 4-methylnitrosamino-1,3pyridyl-1-butanone (nicotine-derived nitrosamine ketone; NNK). The presence of these metabolic products may be correlated with the exposure of the subject to tobacco products. 